\title{
An Investigation into the Impact of COVID-19 on the Relationships Between Parents of Children Diagnosed with Cystic Fibrosis and the Cystic Fibrosis Multi-Disciplinary Team
}

\author{
Steve Jones*, Leanne Smith and Katie Ainsworth \\ Sheffield Children's Hospital NHSFT
}

Pre-press 26 October 2021

Published 28 June 2022

\begin{abstract}
.
Background: The COVID-19 pandemic has seen working practice in Cystic Fibrosis (CF) move to more remote clinical models. This study assesses the impact of shifting working models on the relationships between families of young people with CF and CF Multi-disciplinary Teams (MDT) as well as parents perceptions of working remotely.

Methods: Six semi-structured interviews with parents of young people under the care of a regional UK CF specialist centre were analysed using Inductive content analysis.

Results: Three domains emerged: Interpersonal Relationships, Remote Clinics and CF in the Context of COVID-19. The enduring and close relationships between the MDT and families were discussed as well as the acceptability of remote clinics moving forwards.

Conclusions: The importance of the quality in the relationship between families and CF MDTs is vital to enhance ongoing care. Remote working was acceptable in the context of COVID-19 and with some considerations could be useful moving forwards.
\end{abstract}

Keywords: Cystic fibrosis, MDT, COVID-19, tele-health, psychology, qualitative

\section{INTRODUCTION}

Cystic Fibrosis (CF) is a genetic condition leading to chronic health difficulties throughout an individual's life [1]. In order to maximise their health and life expectancy, individuals diagnosed with CF are supported regularly within CF Multi-disciplinary Teams (MDT). MDTs usually consist of; Doctors, Specialist Nurses, Physiotherapists, Dieticians, Social Workers, Psychologists and Admin staff [2,3].

\footnotetext{
*Correspondence to: Steve Jones, Sheffield Children's Hospital NHSFT. E-mail: stephen.jones3@nhs.net.
}

In the United Kingdom (UK), routine screening programmes mean that $\mathrm{CF}$ is usually diagnosed soon after birth [4]. In line with European guidelines [1], patients are then monitored closely, usually every 6-8 weeks with clinic visits and annual reviews. Inpatient stays for additional medical intervention may also be required. Paediatric CF centres tend to work with young people and their families from diagnosis through to transition into adult services at around 16-18 years old.

CF therefore presents a fairly unique health setting and culture in that patients and families are seen by the same MDT regularly several times a year for many years. Families routinely attend the CF centre 
for clinic visits and inpatient stays where they are seen in turn by members of the same MDT. Previous research suggests that the relationship with the CF MDT is an important factor in adherence to medications, something which is vital to maintaining health status within CF [5]. Therefore, a positive relationship would be considered important, not only due to the number of contacts young people and families have with the CF MDT, but also to enhance their health status.

The recent national and international responses to COVID-19 have led to a shift in models of health care. Individuals diagnosed with $\mathrm{CF}$ are known to be more susceptible to respiratory infections and therefore were advised in the UK to shield to limit their exposure COVID-19 [6]. This had a major impact on how families were able to access their CF teams with respect to less face-to-face contact and a shift to remote clinics and video. While studies have shown that remote clinics can be effective in other countries [7], this is not routine in the UK.

The current study will investigate how the changes in working practice brought about by COVID-19 have impacted on families' relationships with the CF MDT and what this may mean for routine $\mathrm{CF}$ care following the acute phase of COVID-19. The authors have no prior hypothesis regarding the outcomes of this study.

\section{METHOD}

A series of semi-structured interviews were completed to examine the impact of COVID-19 on the relationships between families and a CF MDT. Ethical clearance for this project was granted by the NHS IRAS panel: IRAS-284623.

\section{Participants}

Parents of young people who attended a Regional Paediatric CF centre were approached for their participation in this study by members of the study team (SJ \& LS) as a part of their routine clinic visit on a first-come first-served basis. Given this opportunity sampling method disease severity was not controlled for. If parents expressed interest, then a study information sheet was emailed to them. A follow-up call was conducted a week later to discuss participation, at which point they were free to withdraw or book in an appointment for the interview.

Based on previous research [5], an initial sample of 6 parents were consented for the study. Participants were from across the geographical spread of the Paediatric CF centre covering several counties in the north of England. All participants were female. The average age of the participant's children was 6.7 (range 2-13) with a gender split of 2 females and 5 males (one family had 2 boys diagnosed with $\mathrm{CF}$ ).

Interviews took place in July 2020 at a time when lockdown restrictions were being eased nationally in the UK, yet for some people diagnosed with CF they had been shielding since March 2020 with changes to clinic arrangements having been in place since February.

\section{Semi-structured interview}

Semi-structured interview stem questions were produced a-priori with authors agreeing on the content. Semi-structured interviews provide a flexible structure in order to explore topics in a way that was responsive to the participant's experience. The authors SJ and LS conducted four and two interviews respectively. All interviews were via telephone and recorded on a secure laptop using BigHand software. Interviews lasted on average 30 minutes (range $=16-43$ minutes). Interviews were then transcribed anonymously by KA. Participants were not compensated for their participation.

\section{Data analysis}

Interviews were transcribed verbatim and anonymised by KA before distribution to SJ and LS for analysis. Analysis was conducted using Inductive Content Analysis [8] as has previously been used in this context [5]. SJ and LS read the transcripts in order to immerse themselves within the data before beginning to identify units of meaning or codes within the text. Units of meaning are defined single words or sentences depending upon context but which share a singular thematic meaning. On completion of transcript one's analysis, the authors independently constructed coding manuals detailing themes they identified within the text. The authors then discussed how to synthesise their coding manuals into a coherent structure reflective of the text, taking into account superordinate and subordinate themes. Disagreements were discussed between the authors. A plan to bring in arbitration was agreed if consensus was not reached, however, this was not needed. Following agreement on the initial themes contained within the first coding manual, subsequent transcripts were analysed and incorporated in an iterative manner until a final manual was produced. Data saturation 


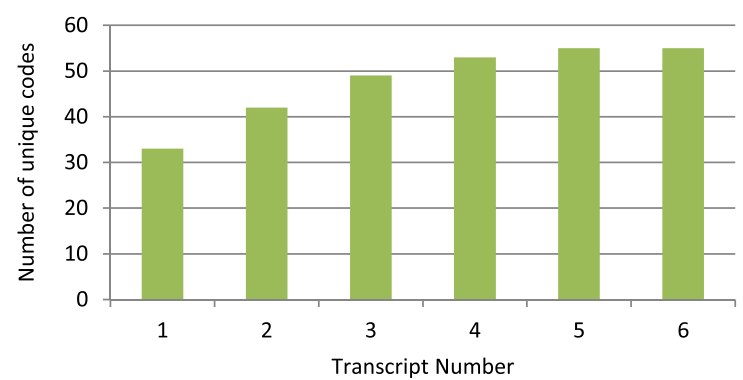

Fig. 1. Cumulative number of unique codes generated following each subsequent transcript's analysis.

was discussed after each subsequent transcript with an agreement being reached following transcript 6 following the discovery of no new codes. Figure 1 demonstrates no new unique codes were found in transcript 6 and a levelling off of new codes generated with each subsequent transcript prior to this.

In order to protect against bias within the interviews and analysis, both authors maintained a reflective diary following each interview. The authors also made time to discuss potential biases together during peer supervision.

\section{RESULTS}

The resultant themes can be split into three main overarching domains: Interpersonal Relationship, Remote Clinics, and CF in the Context of COVID-19. Illustrative quotes for each subordinate theme can be found in Table 1.

\section{Interpersonal relationship}

This domain reflects the qualities of the interpersonal relationship between families and the CF MDT.

\section{Enduring}

Participants described how their relationships with the CF team often started soon after the birth of their child and endured over the course of their child's life. These relationships were said to evolve over time and to develop alongside their child, with a shift to increased child involvement as they aged. Ruptures in the relationship were observed, however, it was noted that ruptures were resolved through discourse. The consistency of the staff team over time allowed for less repetition during consultations as well as an increasing sense of familiarity.

\section{Professional elements}

Commonly, participants commented on the specialist knowledge and expertise of the CF team. Participants discussed how the CF team was their first port of call for questions or concerns about their child whether questions were specifically CF-related or not. The holistic nature of the care provided by the constituent members of the MDT, as well as the approach of putting the child's health as the first priority was also highlighted. Some parents disclosed difficult experiences when working with non-CF specialist medical teams due to a perceived lack of expertise in $\mathrm{CF}$ care.

\section{Personal elements}

All participants discussed the need for trust with the CF team which was facilitated by bi-directional respect and honesty in terms of patient health and needs. Open communication styles and the importance of feeling listened to by a collaborative MDT approach was also valued. Finally, the personal qualities of the MDT with respect to having a friendly, caring and comforting approach, especially during difficult times was important to the overall relationship.

\section{Remote clinics}

Themes within this domain make reference to how remote clinics have positively and negatively impacted upon the care received by families of young people diagnosed with $\mathrm{CF}$ as well as how acceptable this approach may be in the future.

\section{Influence on CF team approach}

The time available for appointments and consultations was said to not reduce following the move to remote clinics, with support being increased via home visits or postal swabs where needed. Often only one person was able to speak and hear the team over the phone which increased the pressure on the individual to remember and discuss information with the team accurately. In contrast, video clinics were said to allow conversations between the CF team and the family which felt more like a traditional face-to-face clinic. Some participants also commented on how remote clinics felt more formal with less opportunity for valued ad-hoc informal chats and questions. The CF team were said to have taken proactive steps to help support families such as providing stocks of antibiotics or having swabs sent out in preparation for not being able to get into clinic in person. 
Table 1

Illustrative quotes obtained from the transcripts of participants

Domain 1: Interpersonal Relationship

Major theme: Enduring
$1.1 \quad \ldots$ we built that relationship really from day one when we had [child's] diagnosis.

$1.2 \ldots$ the relationship is developing with the frequency of appointments so every 6 to 8 weeks we're seen and so over time there's a little bit more familiarity.

1.3 ...I have noticed over the last probably 3 or 4 years that [child] is more involved in the discussion which I think is really important.

1.4 ... we see the same faces 4 years ago what we do today. Having them there, knowing that they're there all the time is so comforting.

\section{Major theme: Professional elements}

2.1 ... they would be my go-to point in the first instance because I trust them and I think they are the experts.

2.2 ... I feel like they are my first port of call when there's anything wrong.

2.3 ... they just seem to know what they are doing. They are on the ball with everything, compared to like every time I use a local service at a local hospital.

2.4 ... because everybody has their different skills I think we pick wisely who will be able to help us the most, erm, and we're not afraid to ask for any support that we need erm, to help [child] as best we can, erm everybody is willing to help and support us and yeah definitely we use everybody who is available to us.

2.5 I don't have any faith in my GP, I've used other hospital services and I have no trust in them because of things that they've done wrong.

2.6 ... like a professional relationship, you know, that we are able to do what's best for your child because they have that bit of distance in terms of, you know you don't have that distance as parents.

Major theme: Personal elements

3.1 But I know the team, I know, I don't know it just, it just feels like they're part of your family.

3.2 ... you just build up that relationship and trust with them and again they're just part of that big family and team that help support us all.

3.3 ... I think they're unique people, the people who care for people with cystic fibrosis, the care and understanding of the condition and what it means for parents and for the child as well, erm, they all care individually, they know the child so well.

3.4 ... time is always taken to explain the reason for any intervention and there's always opportunity for discussion so that as parents we understand erm why a particular intervention is required.

Domain 2: Remote clinics

Major theme: Influence of CF team approach

4.1 ... it was fantastic that the nurses were able to support families by coming out and taking bloods in our homes.

4.2 I suppose the level of care's exactly the same, if not more in that there's been more thought in how they support his care just in a different way, erm so I'd say there's been no reduction at all in the level of care for him.

4.3 ... we still communicate the same, obviously we haven't seen them face to face but they still like communicate through the phone.

4.4 ... I've got to relay that information and I feel like I need to intensively listen otherwise I'll miss something.

Major theme: Advantages

5.1 ... but obviously we have the virus going round, the first thing is you're going to do to reduce the risk of infection is by keeping it on the phone.

5.2 ... the key advantage is that it's a better use of time for families, erm, that you're not having to make provisions for the rest of the family whilst you're at the hospital.

5.3 ... it would actually mean that [child] doesn't have to necessarily be absent from school, erm, he wouldn't be missing half a day of education.

5.4 ... so that's another advantage, so it's less travelling.

\section{Major theme: Disadvantages}

6.1 ... I was really worried about him not having a physical examination.

6.2 ... you get a huge amount of reassurance from a doctor listening to your chest, your child's chest, and nobody can do that unless you're face to face.

6.3 ... the only thing that hasn't happened is his annual review.

6.4 ... the tech a bit unreliable so you know you might drop out of the Wi-Fi or it freezes and you have to go out and come back in.

6.5 I would prefer face to face, I do prefer face to face.

6.6 ... not seeing the guys, like the nurses and doctors, and that like, you know, you do get to have a little chat and then there's the other things that come up when you're there that you probably wouldn't have done over the phone.

Major theme: Acceptability

7.1 I'm happy at the moment that the calls are over the telephone but I think it'd be a different story if I do have concerns about the boy, I think I would then look at a face to face appointment.

7.2 Had it been autumn or winter when he usually has a persistent cough, erm, I would probably have felt a lot more nervous, erm, them not being able to see him face to face.

7.3 ... as long as they get to see him at least like once every say three months then I'll be quite happy to do that.

7.4 I think that they have done the things that are required.

7.5 ... the relationship is almost like a long lost friend who you haven't seen in a long time but you just pick up exactly where you left off last time meeting them face to face. 
Table 1

(Continued)

\section{Domain 3: CF in the context of COVID-19}

Major theme: Routine
$8.1 \quad$...it's just part of our daily routine whatever day it is.

8.2 ... at first there were a lot of practical things to sort out, which also fuels the anxiety, so erm you know organising being able to work at home for my husband, coz he works for the NHS.

\section{Major theme: Emotional impact of the pandemic}

9.1 ... I think at the start of COVID, erm, it was an incredibly anxiety provoking time.

9.2 ... I think I felt very anxious, erm, I felt sick quite a lot of the time and erm you know quite emotional I guess but also trying to balance it with still looking after my family.

9.3 ... it sounds a bit scary like with our life, I don't know, semi-isolation forever, that seems like a scary thought.

$9.4 \ldots$ if [child] developed some Covidy symptoms and lockdown he felt very isolated.

9.5 ... from his mental health I was really concerned, erm, I could see the decline in his loneliness, anger, frustration.

\section{Major theme: Influence on medical care}

10.1 Obviously I was allowed in and whilst she was admitted she wasn't allowed to see her dad, which she found that quite hard

10.2 I think it's been necessary. I think it's reassuring and puts things you know as always how they work to protect our children.

$10.3 \ldots$ in person you get it done there and then and it, you know, erm, with the results I'd usually get a phone call but obviously because you're posting it, and then you're thinking, has it arrived?

\section{Advantages}

Participants described remote clinics saving them time and minimising disruption to their day. Parents required less commuting time for clinics which was a more pleasant experience, especially for those making longer journeys while also reducing travel costs. With clinics taking less time there was also less disruption to education or work. Additionally, participants spoke about the reduced infection risk of remote clinics, especially in the context of the COVID-19 pandemic.

\section{Disadvantages}

Participants explained that a lack of physical examination was a major disadvantage of remote clinics due to reducing confidence that clinicians had been able to fully appreciate their child's health. Parents also spoke about how the less personal remote interaction made it harder to fully absorb information or teaching. It was also noted how remote working was a departure from a routine which parents had confidence in and therefore felt uncomfortable at times. Finally, some parents commented on technical issues with video connections making clinics less streamlined and leading to frustration. Some children were also said to appear less engaged with the team via video as opposed to when visiting them in clinic.

\section{Acceptability}

Parents described feeling that remote clinics were working quite well. Most preferred the enhanced connection offered by video compared to telephone and felt that remote clinics were more acceptable when their child was well but would prefer face-to-face should their child be unwell. To strike a balance between convenience and confidence in their child's health status, some clients suggested alternating remote and face-to-face appointments in the future. The relationship between parents and the CF MDT was said to not be affected too much by working remotely, however, it was mentioned that in the early stages following diagnosis it would be important to keep face-to-face to establish and consolidate the relationship before considering remote working.

\section{CF in the context of COVID-19}

This domain referenced some of the unique challenges that the COVID-19 pandemic has placed upon families under the care of the CF unit. A shift from normal routines and expectations in all areas of life has had an emotional impact upon families.

\section{Routine}

Many parents commented on how precautions such as hand-washing, hygiene and infection control were all a part of their everyday experience and therefore COVID-19 hadn't been too much of a change to daily life. Some parents did however comment that day-today practical issues such as grocery shopping were much more difficult and required additional planning. Most parents spoke of how their child's medication adherence hadn't changed during the pandemic and shielding, however, it was noted that the routine and timings had shifted due to a lack of structure in their day. 


\section{Emotional impacts of the pandemic}

Many parents talked about their anxiety, fear and how COVID-19 may impact upon their child's short and long-term health. Anxiety and worry were noted during the initial wave of the pandemic as well as when families were able to come out of shielding and send their children back into school. Feeling isolated was a theme for many parents and their children who had been in self-isolation longer than their peers which exacerbated feelings of frustration and anger at the situation.

\section{Influence on medical care}

Parents described COVID-19 leading to increased concern about the need for medical admissions given the prevalence of COVID-19 in hospitals. A sense of pragmatism was noted in participants who spoke of getting on with their lives and the adjustments they needed to make in order to support their families and themselves. While accessibility to the CF MDT was said to have remained consistent, families spoke of some of things they weren't able to do as easily due to COVID-19 such as collect medications from the hospital pharmacy or obtain medical supplies.

\section{DISCUSSION}

The COVID-19 pandemic has had a far-reaching impact on health service delivery, especially for $\mathrm{CF}$ patients and their families. There has been a shift from face-to-face clinics to remote working. This study investigated the impact of this shift on the relationships between a Paediatric CF MDT and parents.

The relationship between the CF MDT and its patients is fairly unique in that it endures from the time of diagnosis through to eventual transition into adult service with regular contact roughly every 6-8 weeks [1]. Given the length of this relationship, it is of no surprise that parents spoke of how the relationship endures over time, but also how it evolves to show an increasing involvement of the young person in their own care. A key theme to emerge was the importance of a bi-directional trusting relationship in facilitating effective care. Trust appeared to be related to clinician honesty, respect, perceived expertise, caring, comforting and friendliness. These themes highlight the importance of not only availability and accessibility, but also of the interpersonal qualities required to facilitate enhanced patient care in such a long-standing relationship.
Remote working and tele-health clinics have not previously been routine within this Paediatric CF service, and therefore the current situation presented an organic opportunity to discuss this with parents. The majority of the advantages with remote working were with respect to time and cost savings, especially for those who needed to travel further. Less disruption to daily routines was repeatedly cited as an advantage, especially when considering not needing to miss whole school days for a clinic. Clinicians not being able to physically examine patients was seen as the main disadvantage of remote clinics and was a concern for parents who felt less confident that clinicians had been able to get a full experience of their child's health. It appeared that in times of good or stable health, parents were more accepting of remote clinics but preferred face-to-face clinics should their child's health change. A suggestion put forward was to alternate face-to-face and remote clinics in order to have the advantages of both while minimising the disadvantages.

Parents reported how COVID-19 has led to an increased sense of isolation for families due to shielding. It is worth noting that parents appeared to experience increased anxiety at times of transition such as the beginning of lockdown and school reopening as opposed to frustration or anger in between. Some parents commented on how the pandemic has led the world to act in a way that people with CF diagnoses have always acted and therefore the precautions weren't that different for families despite their being some practical challenges to overcome.

The data highlights the enduring and close relationship between the CF MDT and the parents of young people diagnosed with $\mathrm{CF}$. The move to remote clinics has influenced some of the more informal and ad-hoc interactions between the CF team and the families it supports making the interactions more structured. Despite this, parents reported that there hadn't been a major shift in the relationship, especially in the short-term but it was unknown how this would change over time. Several participants stated how the relationship with the CF team was established long before the changes brought about by COVID-19 and therefore the relationship had a secure base upon which to function. The importance of the face-to-face interactions and how this can help to develop trust in the early stages following a diagnosis of CF were stressed. It is therefore important to consider a balance between remote working reducing infection risk and face-to-face appointments which allow the building of what will be a 
long-standing and important relationship with the family.

At present it is unclear how the health care landscape will continue to be shaped by COVID-19. COVID-19 has led to the rapid advancement of telecommunication software in UK healthcare and has enabled clinicians to begin to deliver remote video clinics in a way which was not possible prior to the pandemic. It is clear parent's perceived that there are some disadvantages to this model of working if used exclusively, however, if used appropriately it may lead to increased satisfaction for clients, especially those who would usually have to travel long distances. Specifically to CF, this technology also presents the opportunity to run group work which often has not been possible due to infection control concerns.

It is difficult to generalise these findings of this study's small sample to a whole cohort of patients, however, the qualitative depth of the information gathered helps to steer research and conversation about enhancing CF care as we begin to emerge from COVID-19. There is also an awareness that the current study focussed on parent's perceptions of the relationship with the CF team. Parents were able to suggest how their child related to the CF MDT and changes in working practice, however; these were suppositions and not direct accounts from the young people themselves. Future development of this work would be to investigate how children of different ages relate to the CF MDT and how this changes over time.

In conclusion, this study allowed insight into parent's experiences of their relationship with the CF MDT and how this has been influenced by COVID19. Suggestions for how remote working can be adapted to support families in the future are suggested and future work may wish to expand on these themes in order to generalise them to the wider Paediatric CF population.

\section{ACKNOWLEDGMENTS}

The authors have no acknowledgments to report.

\section{FUNDING}

The authors have no funding to report.

\section{CONFLICT OF INTEREST}

The authors have no conflict of interest to report.

\section{REFERENCES}

[1] Karem E, Conway S, Elbron S, Heijerman H. Standards of care for patients with cystic fibrosis: A European consensus. Journal of Cystic Fibrosis. 2005;4(1):7-26.

[2] The CF Trust. Standards for the Clinical Care of Children and Adults with Cystic Fibrosis in the UK. 2016. Retrieved online on 23/10/20 from: https://www.cysticfibrosis.org.uk/ / media/documents/the-work-we-do/care/consensus-docswith-new-address/cystic-fibrosis-trust-standards-ofcare. $a \operatorname{ash} x$ ? $1 \mathrm{a}=\mathrm{en}$

[3] Mahadeva R, Webb K, Westerbeek RC, Carroll NR, Dodd ME, Bilton D, Lomas DA. Clinical outcome in relation to care in centres specializing in cystic fibrosis: cross sectional study. BMJ. 1998;316:1771-5.

[4] Schluter DK, Southern KW, Dryden C, Diggle P, TaylorRobinson D. Impact of newborn screening on outcomes and social inequalities in cystic fibrosis: A UK CF registry-based study. Thorax. 2020;75(2):123-31.

[5] Jones S, Babiker N, Gardner E, Royle J, Curley R, Hoo $\mathrm{ZH}$, Wildman MJ. Promoting adherence to nebulized therapy in cystic fibrosis: poster development and a qualitative exploration of adherence. Patient Preference and Adherence. 2015;9:1109-20.

[6] The CF Trust. Statement on how people with cystic fibrosis and their families can help minimise their risk of COVID-19. 2020 Retrieved online on 23/10/20 from: https://www. cysticfibrosis.org.uk/news/uk-cf-medical-associationsstatement-on-coronavirus

[7] Wood J, Mulrennan S, Hill K, Cecins N, Morey S, Jenkins $\mathrm{S}$. Telehealth clinics increase access to care for adults with cystic fibrosis living in rural and remote Western Australia. Journal of Telemedicine and Telecare. 2017;23(7):673-9.

[8] Zhang Y, Wildemuth BM. Qualitative analysis of content. In: Wildemuth BM, editor. Applications of Social Research Methods to Questions in Information and Library Science. Westport, CT: Libraries Unlimited; 2009:308-19. 